\title{
The fractional and mixed-fractional CEV model
}

\author{
Axel A. Araneda* \\ Frankfurt Institute for Advanced Studies \\ 60438 Frankfurt am Main, Germany.
}

This version: June 1, 2019

\begin{abstract}
The continuous observation of the financial markets has identified some 'stylized facts' which challenge the conventional assumptions, promoting the born of new approaches. On the one hand, the long-range dependence has been faced replacing the traditional Gauss-Wiener process (Brownian motion), characterized by stationary independent increments, by a fractional version. On the other hand, the CEV model addresses the Leverage effect and smile-skew phenomena, efficiently. In this paper, these two insights are merging and both the fractional and mixed-fractional extensions for the CEV model, are developed. Using the fractional versions of both the Itô's calculus and the Fokker-Planck equation, the transition probability density function of the asset price is obtained as the solution of a non-stationary Feller process with time-varying coefficients, getting an analytical valuation formula for a European Call option. Besides, the Greeks are computed and compared with the standard case.
\end{abstract}

Keywords: fBM, mfBm, CEV, fractional Fokker-Planck, fractional Itô's calculus, Feller's process

\section{Introduction}

One of the most important insights in financial mathematics has been the Black-Scholes model [1, which uses a Geometric Brownian motion (GBM) for describes the returns of the asset prices as a regular diffusion process and arriving at an analytical formula for a Vanilla European option.

However, some "Stylized facts" in the financial markets don't agree with the assumptions using in the Black-Scholes model. One of these findings is the long-range dependence 2 25], motivating the creation of a fractal version of the Black-Scholes model [6, 7, based on fractional Brownian motion 8 10]. Hu \& Øskendal 11] and Necula [12] arrive at an analytical formula for the European Call option for the fractional Black-Scholes case, using Wick-Itô calculus [13, 14]. Nonetheless, in the fractional framework, the arbitrage possibilities aren't enterely omitted [15-17. Addressing it fact, Cheridito [18 introduces the mixed-fractional Brownian motion (see further mathematical details at refs. [19, 20]). This kind of model ensures the absence of arbitrage opportunities 21, 22, and also a pricing formula for European type contracts could be obtained [23, 24].

On the other hand, and come back to shortcomings of the original Black-Scholes model, the homoscedasticity assumption is not consistent with other empirical facts as the volatility smile-skew [25]28] and leverage effect 29 32]. The former is the change in the implied volatility pattern as a function of the strike price of an option. The latter is understood as the inverse relationship between the volatility and the price. In this context, a very popular formulation is the Constant Elasticity of Variance (CEV) model developed by Cox [33, 34, which faces the heteroscedasticity and the leverage effect modeling the volatility as a function of the asset price level. The model also deals with the skew-smile phenomena 35, 36. Despite that the CEV model considers only one more parameter (elasticity) than the BlackScholes mode ${ }^{2}$, the latter is outperformed by the CEV in both prices and option pricing performance 38 41. Another plus point to the use of the CEV model is existence of a closed form formula for a European vanilla option. The original Cox's work derives the Call price in terms of summations of the incomplete gamma function, but later Schroder [42] developed a closed-form solution depending on the

\footnotetext{
*Email: araneda@fias.uni-frankfurt.de, Tel.:+49 6979847501

1 a.k.a persistence, 'Memory effect' or 'Joseph effect'

${ }^{2}$ See [37] to details on the parameter estimation issue under CEV.
} 
non-central $\chi^{2}$ distribution ${ }^{3}$, See Refs. [45 47] for recent and successfully applications of the CEV model in different contexts.

Given the previous statement, the aim of this paper is to merge the local volatility approach and the fractional calculus, extending the CEV model under classical Brownian motion to the fractional and mixed-fractional cases. The fractional CEV case has been addressed previously in the literature [48, proposing a European Call formula in terms of the standard complementary gamma distribution function, similar to the Cox's result, but without explicit evaluation of the added terms. This time, for the fractional CEV, the European Call price is derived by a compact and explicit way, in terms of the non-central-chi-squared distribution and the M-Whittaker function, following the Schroder scheme and using a time-varying coefficients' version of the Feller's diffusion problem. Besides, similarly, a pricing formula for the mixed-fractional CEV model is studied. Also, the convergence of the fractional CEV pricing to the fractional Black-Scholes case is shown. Moreover, the Greeks of the models are computed and compared with the standard CEV case.

The paper outline is the following. First, the CEV model is revisited. Later, the fractional extension is analyzed, deriving the pricing formula for a European Call option. After that, a mixed fractional structure is proposed, arriving at the related European Call pricing formula. At next, the computation and analysis of the Greeks, are performed. Finally, the main conclusions are displayed.

\section{The CEV model}

Under the risk-neutral measure, at the constant elasticity of variance model, the asset price $S$ follows the next stochastic differential equation:

$$
\mathrm{d} S=r S \mathrm{~d} t+\sigma S^{\frac{\alpha}{2}} \mathrm{~d} B_{t}
$$

where $r, \sigma$ and $\alpha$ are the constant parameters of the model, with $\sigma>0$ and $\alpha \in\left[0,2\left[. B_{t}\right.\right.$ is a standard Brownian motion, such that $\mathrm{d} B_{t} \sim N(0, \mathrm{~d} t)$. In the limit case, when $\alpha \rightarrow 2$, the CEV turns into the Black-Scholes model.

Aplying the following change of variable:

$$
x(S, t)=S^{2-\alpha}
$$

and by the Itô's Lemma, Eq. (1) becomes:

$$
\mathrm{d} x=(2-\alpha)\left[r x+\frac{1}{2}(1-\alpha) \sigma^{2}\right] \mathrm{d} t+(2-\alpha) \sigma \sqrt{x} \mathrm{~d} B_{t}
$$

Let $P\left(x_{T}, T \mid x_{0}, 0\right)$, the transition probability function which rules the evolution of $x$ from $x(0)=x_{0}$ to $x(T)=x_{T}$, and $T>0$. Then, $P$ evolves according to the Fokker-Planck equation:

$$
\frac{\partial P}{\partial t}=\frac{1}{2} \frac{\partial^{2}}{\partial x^{2}}\left[(2-\alpha)^{2} \sigma^{2} x P\right]-\frac{\partial}{\partial x}\left[(2-\alpha)\left(r x+\frac{1}{2}(1-\alpha) \sigma^{2}\right) P\right]
$$

Eq. (3) can be solved by the famous Feller's lemma [49, summarized at next:

Feller's lemma. Let $u=u(x, t)$, and $a, b, c$ constants, with $a>0$ and $t>0$. The solution of the parabolic equation

\footnotetext{
${ }^{3}$ The computation of the non-central chi-squared distribution in Schroeder's formula is quite unstable and becomes expensive for elasticities near to zero. In order to reduce the computational times and also to address American-type options, several analytical approximations and numerical methods have been developed for the CEV model, see [43, 44] for a survey.
} 


$$
\frac{\partial u}{\partial t}=\frac{\partial^{2}}{\partial x^{2}}[a x u]-\frac{\partial}{\partial x}[(b x+c) u]
$$

conditional to

$$
u(x, 0)=\delta\left(x-x_{0}\right)
$$

is given by

$$
u\left(x, t \mid x_{0}, 0\right)=\frac{b}{a\left(e^{b t}-1\right)}\left(\frac{x e^{-b t}}{x_{0}}\right)^{\frac{c-a}{2 a}} \exp \left[-\frac{b\left(x+x_{0} e^{b t}\right)}{a\left(e^{b t}-1\right)}\right] I_{1-c / a}\left[\frac{2 b}{a\left(1-e^{-b t}\right)} \sqrt{e^{-b t} x_{0} x}\right]
$$

where $I_{k}(x)$ is the modified Bessel function of the first kind of order $k$.

Proof. See Refs. [49, [50].

If we set $a=(2-\alpha)^{2} \sigma^{2} / 2, b=r(2-\alpha)$ and $c=(2-\alpha)(1-\alpha) \sigma^{2} / 2$; by the Feller's Lemma the transition probability distribution from $x(0)=x_{0}$ to $x(T)=x_{T}$, is given by:

$$
\begin{aligned}
P\left(x_{T}, T \mid x_{0}, 0\right)=\frac{2 r}{\sigma^{2}(2-\alpha)\left[\mathrm{e}^{r(2-\alpha) T}-1\right]} & \left(\frac{x_{T}}{x_{0}} \mathrm{e}^{-r(2-\alpha) T}\right)^{-\frac{1}{2(2-\alpha)}} \exp \left[-\frac{2 r\left(x+x_{0} \mathrm{e}^{r(2-\alpha) T}\right)}{\sigma^{2}(2-\alpha)\left(\mathrm{e}^{r(2-\alpha) T}-1\right)}\right] \\
& \times I_{1 /(2-\alpha)}\left[\frac{4 r}{\sigma^{2}(2-\alpha)\left(1-\mathrm{e}^{-r(2-\alpha) T}\right)} \sqrt{\mathrm{e}^{-r(2-\alpha) T} x_{0} x}\right]
\end{aligned}
$$

Coming back to the original variables and reordering terms, the density for $S_{T}$ given $S_{0}$ is equal to 42]:

$$
\begin{aligned}
P\left(S_{T}, T \mid S_{0}, 0\right) & =P\left(x_{T}, T \mid x_{0}, 0\right) \frac{\partial x_{T}}{\partial S_{T}} \\
& =(2-\alpha) k^{\frac{1}{2-\alpha}}\left(y w^{1-2 \alpha}\right)^{\frac{1}{2(2-\alpha)}} \mathrm{e}^{-y-w} I_{1 /(2-\alpha)}(2 \sqrt{y w})
\end{aligned}
$$

where:

$$
\begin{aligned}
k & =\frac{2 r}{\sigma^{2}(2-\alpha)\left[\mathrm{e}^{r(2-\alpha) T}-1\right]}, \\
y & =k S_{0}^{2-\alpha} \mathrm{e}^{r(2-\alpha) T}, \\
w & =k S_{T}^{2-\alpha} .
\end{aligned}
$$

Later, the value of a Call option at time $t=0$, with maturity $T$ and exercise price $E$, is computed by the Feynman-Kac formula: 


$$
\begin{aligned}
C(S, 0)= & \mathrm{e}^{-r T} \int_{-\infty}^{\infty} \max \left\{S_{T}-E, 0\right\} P\left(S_{T}, T \mid S_{0}, 0\right) \mathrm{d} S_{T} \\
= & \mathrm{e}^{-r T} \int_{E}^{\infty}\left(S_{T}-E\right) P\left(S_{T}, T \mid S_{0}, 0\right) \mathrm{d} S_{T} \\
= & \mathrm{e}^{-r T} \int_{z}^{\infty}\left[\left(\frac{w}{k}\right)^{\frac{1}{2-\alpha}}-E\right](2-\alpha) k^{\frac{1}{2-\alpha}}\left(y w^{1-2 \alpha}\right)^{\frac{1}{2(2-\alpha)}} \mathrm{e}^{-y-w} \\
& \times I_{1 /(2-\alpha)}(2 \sqrt{y w})\left[\frac{1}{2-\alpha}\left(k w^{1-\alpha}\right)^{-\frac{1}{2-\alpha}}\right] \mathrm{d} w \\
= & \mathrm{e}^{-r T} \int_{z}^{\infty}\left(\frac{w}{k}\right)^{\frac{1}{2-\alpha}}\left(\frac{y}{w}\right)^{\frac{1}{2(2-\alpha)}} \mathrm{e}^{-y-w} I_{1 /(2-\alpha)}(2 \sqrt{y w}) \mathrm{d} w \\
& -\mathrm{e}^{-r T} \int_{z}^{\infty} E\left(\frac{y}{w}\right)^{\frac{1}{2(2-\alpha)}} \mathrm{e}^{-y-w} I_{1 /(2-\alpha)}(2 \sqrt{y w}) \mathrm{d} w \\
= & C_{1}-C_{2}
\end{aligned}
$$

where $z=k E^{2-\alpha}$. As pointed by Schroder [42, the arguments of both integrals are the pdfs of the non-central chi-squared distributions with $\nu$ degrees of freedom and non-centrality parameter $\lambda$, noted by $\chi_{\nu}^{2}(\lambda)$ and defined as:

$$
\begin{aligned}
P_{\chi_{\nu}^{2}(\lambda)}(l) & =\left(\frac{x}{\lambda}\right)^{\frac{\nu-2}{4}} \mathrm{e}^{-(x+\lambda) / 2} I_{\nu}(\sqrt{x \lambda}) \\
& =f(l ; \nu, \lambda)
\end{aligned}
$$

Back to the pricing equation, the first integral is developed as:

$$
\begin{aligned}
C_{1} & =\mathrm{e}^{-r T} \int_{z}^{\infty} k^{-\frac{1}{2-\alpha}}(w y)^{\frac{1}{2(2-\alpha)}} \mathrm{e}^{-y-w} I_{1 /(2-\alpha)}(2 \sqrt{y w}) \mathrm{d} w \\
& =\mathrm{e}^{-r T} \int_{z}^{\infty}\left(\frac{y}{k}\right)^{\frac{1}{2-\alpha}}(w / y)^{\frac{1}{2(2-\alpha)}} \mathrm{e}^{-y-w} I_{1 /(2-\alpha)}(2 \sqrt{y w}) \mathrm{d} w \\
& =\mathrm{e}^{-r T} \int_{z}^{\infty}\left(S_{0} \mathrm{e}^{r T}\right)(w / y)^{\frac{1}{2(2-\alpha)}} \mathrm{e}^{-y-w} I_{1 /(2-\alpha)}(2 \sqrt{y w}) \mathrm{d} w \\
& =S_{0} \int_{z}^{\infty}(2 w / 2 y)^{\frac{1}{2(2-\alpha)}} \mathrm{e}^{-(2 y+2 w) / 2} I_{2+\frac{2}{2-\alpha}}(\sqrt{(2 y)(2 w)}) \mathrm{d} w \\
& =S_{0} \int_{z}^{\infty} f\left(2 w, 2+\frac{2}{2-\alpha}, 2 y\right) \mathrm{d} w \\
& =S_{0} \int_{z}^{\infty} f\left(2 w, 2+\frac{2}{2-\alpha}, 2 y\right) \mathrm{d} w
\end{aligned}
$$

While the second one:

$$
\begin{aligned}
C_{2} & =E \mathrm{e}^{-r T} \int_{z}^{\infty}\left[\frac{2 y}{2 w}\right]^{\frac{1}{2(2-\alpha)}} \mathrm{e}^{-(2 y-2 w) / 2} I_{2+\frac{2}{2-\alpha}}(\sqrt{(2 y)(2 y)}) \mathrm{d} w \\
& =E \mathrm{e}^{-r T} \int_{z}^{\infty} f\left(2 y, 2+\frac{2}{2-\alpha}, 2 w\right) \mathrm{d} w
\end{aligned}
$$

Called $Q$ to the complementary distribution function of $\chi_{\nu}^{2}(\lambda)$ : 


$$
\int_{m}^{\infty} f(l ; \nu, \lambda) \mathrm{d} l=Q(m, \nu, \lambda)
$$

and using the following identity [42]:

$$
\int_{m}^{\infty} f(2 l ; 2 \nu, 2 \lambda) \mathrm{d} \lambda=1-Q(2 l ; 2 \nu-2,2 m)
$$

the call formula can be wrote as:

$$
C(S, 0)=S_{0} Q\left(2 z ; 2+\frac{2}{2-\alpha}, 2 y\right)-E \mathrm{e}^{-r T}\left[1-Q\left(2 y ; \frac{2}{2-\alpha}, 2 z\right)\right]
$$

As we remarked previously, the Black-Scholes model can be treated as a limit case of the CEV model, when $a \rightarrow 2$. For observe the convergence of the solution given in 111) to the Black-Scholes case, we will use the following result for the complementary distribution function $Q$, based on the central limit theorem [51:

$$
Q(m, \nu, \lambda) \approx Q_{N}\left(\frac{m-(\nu+\lambda)}{\sqrt{2(\nu+2 \lambda)}}\right), \text { as } \nu \rightarrow \infty
$$

where $Q_{N}(\cdot)$ is the standard normal complementary density function.

Thus, the first complementary function of the Eq. (11), when $\alpha \rightarrow 2$, is computed as:

$$
\begin{aligned}
\lim _{a \rightarrow 2^{-}} Q\left(2 z ; 2+\frac{2}{2-\alpha}, 2 y\right)= & Q_{N}\left[\lim _{a \rightarrow 2^{-}} \frac{2 z-2-\frac{2}{2-\alpha}-2 y}{\sqrt{2\left(2+\frac{2}{2-\alpha}+4 y\right)}}\right] \\
= & Q_{N}\left[\lim _{a \rightarrow 2^{-}} \frac{2 r E^{2-\alpha}-2 r S_{0}^{2-\alpha} \mathrm{e}^{r T(2-\alpha)}+\sigma^{2}(3-\alpha)\left(\mathrm{e}^{r T(2-\alpha)}-1\right)}{\sqrt{\sigma^{2}(2-\alpha)\left(\mathrm{e}^{r T(2-\alpha)}-1\right)}}\right. \\
& \left.\times \frac{1}{\sqrt{4 r S_{0}^{2-\alpha} \mathrm{e}^{r T(2-\alpha)}+\sigma^{2}(3-\alpha)\left(\mathrm{e}^{r T(2-\alpha)}-1\right)}}\right] \\
= & Q_{N}\left[-\frac{\ln \left(\frac{S_{0}}{E}\right)+\left(r+\frac{1}{2} \sigma^{2}\right) T}{\sigma \sqrt{t}}\right] \\
= & Q_{N}\left(-d_{1}\right)
\end{aligned}
$$

and for the symmetry of the normal function, we have that:

$$
Q_{N}(-d 1)=N\left(d_{1}\right)
$$

being $N(\cdot)$ the standard normal cumulative density.

The calculus of second $Q$ function of the Eq. [11), when the degrees of freedom tends to infinity, is: 


$$
\begin{aligned}
\lim _{a \rightarrow 2^{-}} Q\left(2 y ; \frac{2}{2-\alpha}, 2 z\right)= & Q_{N}\left[\lim _{a \rightarrow 2^{-}} \frac{2 y-\frac{2}{2-\alpha}-2 x}{\sqrt{2\left(\frac{2}{2-\alpha}+4 x\right)}}\right] \\
= & Q_{N}\left[\lim _{a \rightarrow 2^{-}} \frac{2 r S_{0}^{2-\alpha} \mathrm{e}^{r T(2-\alpha)}-2 r E^{2-\alpha}-2 \sigma^{2}\left(\mathrm{e}^{r T(2-\alpha)}-1\right)}{\sqrt{\sigma^{2}(2-\alpha)\left(\mathrm{e}^{r T(2-\alpha)}-1\right)}}\right. \\
& \left.\times \frac{1}{\sqrt{4 r E^{2-\alpha}+\sigma^{2}\left(\mathrm{e}^{r T(2-\alpha)}-1\right)}}\right] \\
= & Q_{N}\left[\frac{\ln \left(\frac{S_{0}}{E}\right)+\left(r-\frac{1}{2} \sigma^{2}\right) T}{\sigma \sqrt{t}}\right] \\
= & Q_{N}\left(d_{2}\right)
\end{aligned}
$$

and for the identity between de cumulative and complementary function:

$$
1-Q_{N}(d 2)=N\left(d_{2}\right)
$$

Later, at the limit $\alpha \rightarrow 2$, the European Call pricing of the CEV model converges to:

$$
\lim _{a \rightarrow 2^{-}} C(S, 0)=S_{0} N\left(d_{1}\right)-E \mathrm{e}^{-r T} N\left(d_{2}\right)
$$

which is the classical Black-Scholes formula provided in [1].

\section{A fractional CEV model}

Now, to address the 'Joseph effect' at the CEV environment, the standard Brownian motion of the Eq. (1) is switched by a fractional on $4^{4}{ }^{5}{ }^{6}$ :

$$
\mathrm{d} S=r S \mathrm{~d} t+\sigma S^{\frac{\alpha}{2}} \mathrm{~d} B_{t}^{H}
$$

where $B_{t}^{H}$ is a fractional Brownian motion with Hurst exponent $H>1 / 2$.

Considering the shift of coordinates defined in Eq. 22] and the fractional Itô's formula [54, 55], the Eq. 15 changes to:

$$
\mathrm{d} x=(2-\alpha)\left[r x+H t^{2 H-1}(1-\alpha) \sigma^{2}\right] \mathrm{d} t+(2-\alpha) \sigma \sqrt{x} \mathrm{~d} B_{t}
$$

\footnotetext{
${ }^{4}$ Following [13], a fBM is a Gaussian process which fulfills (for $0<H<1 ; t, s \geq 0$ ):

i) $\mathbb{E}\left(B_{t}^{H}\right)=0$

ii) $\mathbb{E}\left(B_{t}^{H} \cdot B_{s}^{H}\right)=\frac{1}{2}\left\{|t|^{2 H}+|s|^{2 H}-|t-s|^{2 H}\right\}$

Then, for $H>1 / 2$, the autocorrelation function of $B_{t}^{H}$ is positive and decays hyperbolically in function of the lags, i.e., long range dependency: $\sum_{n=1}^{\infty} \mathbb{E}\left[B_{1}^{H} \cdot\left(B_{n+1}^{H}-B_{n}^{H}\right)\right]=\infty$.

${ }^{5}$ Analogously to their classical counterpart, by the fractional Girsanov theorem (see 11 12, 52]), Eq. 15 is wrote under the risk-neutral $\mathbb{Q}$-measure with drift $r$, where $B_{H}^{t}$ is a $\mathbb{Q}$-fractional Brownian motion.

${ }^{6}$ The fractional Brownian motion is not a semi-martingale for $H \neq 1 / 2$; i.e., there is not an equivalent martingale measure. As pointed in [53, and despite the non-martingale condition, the $\mathbb{Q}$-expected discounted value is equal to the current value.
} 
Then, the fractional Fokker-Planck equation [56 [58] related to the stochastic process defined in Eq. (16), is given by:

$$
\begin{aligned}
\frac{\partial P_{H}}{\partial t} & =\frac{\partial^{2}}{\partial x^{2}}\left[H t^{2 H-1}(2-\alpha)^{2} \sigma^{2} x P\right]-\frac{\partial}{\partial x}\left[(2-\alpha)\left(r x+H t^{2 H-1}(1-\alpha) \sigma^{2}\right) P\right] \\
& =\frac{1}{2} \frac{\partial^{2}}{\partial x^{2}}\left[2 H t^{2 H-1}(2-\alpha)^{2} \sigma^{2} x P\right]-\frac{\partial}{\partial x}\left[(2-\alpha)\left(r x+2 H t^{2 H-1} \frac{1}{2}(1-\alpha) \sigma^{2}\right) P\right]
\end{aligned}
$$

Unfortunately, the relation (17) can't be solved using the Feller's lemma because the coefficient are time-dependent (i.e, non-constant). However, Masoliver [59] provides an interesting approach for the non-stationary Feller process when the coefficients are time-dependent, and the main useful result for us is provided in the following statement:

Feller's lemma with time-varying coefficients. Let $u=u(x, \tau), A=A(\tau), C=C(\tau)$ and $\theta$ constant defined by

$$
\theta=\frac{C(\tau)}{A(\tau)}
$$

The solution of the parabolic equation

$$
\frac{\partial u}{\partial \tau}=\frac{\partial^{2}}{\partial x^{2}}[A x u]+\frac{\partial}{\partial x}[(x-C) u]
$$

conditional to

$$
u(x, 0)=\delta\left(x-x_{0}\right)
$$

is given by

$$
u\left(x, \tau \mid x_{0}, 0\right)=\frac{1}{\phi(\tau)}\left(\frac{x e^{\tau}}{x_{0}}\right)^{\frac{\theta-1}{2}} \exp \left[-\frac{\left(x+x_{0} e^{-\tau}\right)}{\phi(\tau)}\right] I_{1-\theta}\left[\frac{2}{\phi(\tau)} \sqrt{e^{\tau} x_{0} x}\right]
$$

where $I_{k}(x)$ is the modified Bessel function of the first kind of order $k$ and

$$
\phi(\tau)=\int_{0}^{\tau} A(\tau-s) e^{-s} d s
$$

Proof. See Ref. [59].

So, if we use the previous definitions of $a, b, c$ (cf. page 3), and set:

$$
\begin{aligned}
\tau & =-b t \\
A(\tau) & =-\frac{a}{b} 2 H\left(-\frac{\tau}{b}\right)^{2 H-1} \\
C(\tau) & =-\frac{c}{b} 2 H\left(-\frac{\tau}{b}\right)^{2 H-1}
\end{aligned}
$$

Eq. (17) transforms into:

$$
\frac{\partial P_{H}}{\partial \tau}=\frac{\partial^{2}}{\partial x^{2}}[A(\tau) x P]+\frac{\partial}{\partial x}[(x+C(\tau)) P]
$$

and $\theta=C(\tau) / A(\tau)=c / a$, a constant.

Then, Eq. (21), can be solved by the Feller's lemma with time-varying coefficients. Indeed: 


$$
P_{H}\left(x, \tau \mid x_{0}, 0\right)=\frac{1}{\phi(\tau)}\left(\frac{x \mathrm{e}^{\tau}}{x_{0}}\right)^{\frac{c-a}{2 a}} \exp \left[-\frac{\left(x+x_{0} \mathrm{e}^{-\tau}\right)}{\phi(\tau)}\right] I_{1-c / a}\left[\frac{2}{\phi(\tau)} \sqrt{\mathrm{e}^{-\tau} x_{0} x}\right]
$$

where:

$$
\begin{aligned}
\phi(\tau) & =-\frac{a}{b} \int_{0}^{\tau} 2 H\left(\frac{s-\tau}{b}\right){ }^{2 H-1} \mathrm{e}^{-s} \mathrm{~d} s \\
& =\frac{a}{2 H+1}\left(-\frac{\tau}{b}\right)^{2 H}\left[2 H+1+\mathrm{e}^{-\frac{1}{2} \tau}(-\tau)^{-H} M_{H, H+1 / 2}(-\tau)\right]
\end{aligned}
$$

and $M_{\kappa, v}(l)$ is the M-Whittaker function 763 65] and can be expressed in terms of the $M$ confluent hypergeometric Kummer's function:

$$
M_{\kappa, v}(l)=l^{v+1 / 2} \mathrm{e}^{-l / 2} M\left(v-\kappa+\frac{1}{2}, 1+2 v ; l\right)
$$

Now, solving for the original time-coordinate, at time $t=T$, we have:

$$
P_{H}\left(x, T \mid x_{0}, 0\right)=\frac{1}{\phi(T)}\left(\frac{x \mathrm{e}^{-b T}}{x_{0}}\right)^{\frac{c-a}{2 a}} \exp \left[-\frac{\left(x+x_{0} \mathrm{e}^{b T}\right)}{\phi(\tau)}\right] I_{1-c / a}\left[\frac{2}{\phi(T)} \sqrt{\mathrm{e}^{b T} x_{0} x}\right]
$$

with

$$
\phi(T)=\frac{a}{2 H+1} T^{2 H}\left[2 H+1+\mathrm{e}^{\frac{1}{2} b T}(b T)^{-H} M_{H, H+1 / 2}(b T)\right]
$$

Later, moving to the original frame of reference $(S, t)$, and replacing the values for $a, b$ and $c$, the probability density function of $S(T)=S_{T}, T>0$, given $S(0)=S_{0}$ is:

$$
\begin{aligned}
P_{H}\left(S_{T}, T \mid S_{0}, 0\right) & =P_{H}\left(x_{T}, T \mid x_{0}, 0\right) \frac{\partial x_{T}}{\partial S_{T}} \\
& =(2-\alpha) k_{H}^{\frac{1}{2-\alpha}}\left(y w^{1-2 \alpha}\right)^{\frac{1}{2(2-\alpha)}} \mathrm{e}^{-y-w} I_{1 /(2-\alpha)}(2 \sqrt{y w})
\end{aligned}
$$

being:

$$
\begin{aligned}
k_{H} & =[\phi(T)]^{-1}, \\
y_{H} & =k_{H} S_{0}^{2-\alpha} \mathrm{e}^{r(2-\alpha) T} \\
w_{H} & =k_{H} S_{T}^{2-\alpha}
\end{aligned}
$$

\footnotetext{
${ }^{7}$ The Whittaker (or confluent hypergeometric) function appears in the solution of other related problems that involve the CEV process, see for example [60,62], among others.
} 
The transition probabilities $P$ (Eq. (5) and $P_{H}$ (Eq. 222) differ only by the terms $k$ and $k_{H}$. For the particular case $H=1 / 2$, these terms are equa $\left.\right|^{8}$, yield to $P=\left.P_{H}\right|_{H=1 / 2}$.

After that, a European option price may be computed taking expectations of the discounted payoff (see appendix A). Fixing $z_{H}(t)=k_{H}(t) E^{2-\alpha}$, and following the development given from the Eq. 95 to the Eq. 11p, the pricing for a European call, in the fractal CEV, becomes:

$$
C_{H}\left(S_{0}, 0\right)=S_{0} Q\left(2 z_{H} ; 2+\frac{2}{2-\alpha}, 2 y_{H}\right)-E \mathrm{e}^{-r T}\left[1-Q\left(2 y_{H} ; \frac{2}{2-\alpha}, 2 z_{H}\right)\right]
$$

Using the derivatives, asymptotics and recurrence properties ${ }^{9}$ of both $M_{\kappa, v}(l)$ and $M$ [65], from (26) an interesting result is obtained computing the limit case $\alpha \rightarrow 2$. By Eq. 12, we get:

$$
\begin{aligned}
\lim _{\alpha \rightarrow 2^{-}} Q\left(2 z_{H} ; 2+\frac{2}{2-\alpha}, 2 y_{H}\right) & =Q_{N}\left[\lim _{a \rightarrow 2^{-}} \frac{2 z_{H}-2-\frac{2}{2-\alpha}-2 y_{H}}{\sqrt{2\left(2+\frac{2}{2-\alpha}+4 y_{H}\right)}}\right] \\
& =Q_{N}\left[\lim _{a \rightarrow 2^{-}} \frac{E^{2-a l p h a}-\phi(T)\left(1+\frac{1}{2-\alpha}\right)-S_{0}^{2-\alpha} \mathrm{e}^{(2-\alpha) T}}{\sqrt{\phi(T)} \sqrt{\phi(T)\left(1+\frac{1}{2-\alpha}\right)+2 S_{0}^{2-\alpha} \mathrm{e}^{(2-\alpha) T}}}\right] \\
& =Q_{N}\left[-\frac{\ln \left(\frac{S_{0}}{E}\right)+r T+\frac{1}{2} \sigma^{2} T^{2 H}}{\sigma \sqrt{T^{2 H}}}\right] \\
& =Q_{N}\left(-d_{1}^{H}\right) \\
& =N\left(d_{1}^{H}\right)
\end{aligned}
$$

${ }^{9}$ Mainly, we use:

$$
\begin{aligned}
M_{1 / 2,1}(l)=\frac{2 \mathrm{e}^{-l / 2}\left[\mathrm{e}^{l}-(l+1)\right]}{\sqrt{l}} & \Longrightarrow \mathrm{e}^{l / 2}(l)^{-1 / 2} M_{1 / 2,1}(l)=\frac{2}{l}\left[\mathrm{e}^{l}-(l+1)\right] \\
& \Longrightarrow \frac{l}{2 b}\left[2+\mathrm{e}^{l / 2}(l)^{-1 / 2} M_{1 / 2,1}(l)\right]=\frac{1}{b}\left(\mathrm{e}^{l}-1\right) \\
& \left.\Longrightarrow \phi(t)\right|_{H=1 / 2}=\frac{a}{b}\left(\mathrm{e}^{b t}-1\right) \\
& \left.\Longrightarrow k_{H}\right|_{H=1 / 2}=\frac{b}{a\left(\mathrm{e}^{b T}-1\right)}=k
\end{aligned}
$$

- $\lim _{l \rightarrow 0} M_{\kappa, v}(l)=0$

- $\frac{\partial}{\partial l} M_{\kappa, v}(l)=\left(\frac{1}{2}-\frac{\kappa}{l}\right) M_{\kappa, v}(l)+l^{-1}\left(\frac{1}{2}+\kappa+v\right) M_{\kappa+1, v}(l)$

- $M_{\kappa, \kappa+1 / 2}(l)+M_{\kappa+1, \kappa+1 / 2}(l)=M_{\kappa, \kappa+1 / 2}(l)+l^{\kappa+1} \mathrm{e}^{-l / 2}$ 


$$
\begin{aligned}
\lim _{\alpha \rightarrow 2^{-}} Q\left(2 y_{H} ; \frac{2}{2-\alpha}, 2 z_{H}\right) & =Q_{N}\left[\lim _{a \rightarrow 2^{-}} \frac{2 y_{H}-\frac{2}{2-\alpha}-2 z_{H}}{\sqrt{2\left(\frac{2}{2-\alpha}+4 z_{H}\right)}}\right] \\
& =Q_{N}\left[\lim _{a \rightarrow 2^{-}} \frac{S_{0}^{2-\alpha} \mathrm{e}^{(2-\alpha) T}-E^{2-a l p h a}-\phi(T)\left(\frac{1}{2-\alpha}\right)}{\sqrt{\phi(T)} \sqrt{\phi(T)\left(\frac{1}{2-\alpha}\right)+2 E_{0}^{2-\alpha}}}\right] \\
& =Q_{N}\left[\frac{\ln \left(\frac{S_{0}}{E}\right)+r T-\frac{1}{2} \sigma^{2} T^{2 H}}{\sigma \sqrt{T^{2 H}}}\right] \\
& =Q_{N}\left(d_{2}^{H}\right) \\
& =1-N\left(d_{2}^{H}\right)
\end{aligned}
$$

and replacing in (26), we arrive at the fractional Black-Scholes formula [11, 12]:

$$
C_{H}(S, 0)=S_{0} N\left(d_{1}^{H}\right)-E \mathrm{e}^{-r T} N\left(d_{2}^{H}\right)
$$

Then, the convergence of the CEV to the Black-Scholes model, in the limit case $\alpha \rightarrow 2$, remains in the fractional scheme.

Fig. 1 plots the values of the fractional CEV formula for $\sigma$ equal to $15 \%$ (blue) and $30 \%$ (red), and three values of $H=\{0.5,0.7,0.9\}$ considering different maturities. The solid lines draw the case $H=1 / 2$, which corresponds to the classical CEV pricing. The semi-solid and dotted lines show the pricing using $H=0.7$ and $H=0.9$, respectively. The fractional CEV retains the property of being a monotonically increasing function of the elasticity parameter. Also, is a rising function of $\sigma$ and $T$. For expiration times below the year (Figs. 1a 1b), the option price falls if $H$ moves to 1 . In the opposite way, for $T>1$ (Figs. $1 \mathrm{c}[\mathrm{dd}$, the prices grow if $H$ rise in the interval $[1 / 2,1[$. When $\alpha=2$, the fractional CEV pricing transforms into the fractional Black-Scholes price.

\section{A mixed-fractional CEV model}

A mixed-fractional Brownian motion, is defined as a linear combination between an standard Brownian motion and other independent and fractional Brownian motion ${ }^{10}$

$$
M_{t}^{\beta, \gamma, H}=\beta B_{t}+\gamma B_{t}^{H} ; \quad \beta \geq 0, \gamma \geq 0,
$$

Then, for to extent the CEV model to the mixed-fractional case, the Brownian motion which drives the CEV model is replaced by $M_{t}^{H, \beta}$ :

$$
\begin{aligned}
\mathrm{d} S & =r S \mathrm{~d} t+\sigma S^{\frac{\alpha}{2}} \mathrm{~d} M_{t}^{\beta, \gamma, H} \\
& =r S \mathrm{~d} t+\sigma S^{\frac{\alpha}{2}}\left(\beta \mathrm{d} B_{t}+\gamma \mathrm{d} B_{t}^{H}\right)
\end{aligned}
$$

Is clear that if $(\beta, \gamma)=(0,1)$ we recover the fractional case studied in the previous section. Also, if $\beta=1$ and $\gamma=0$, Eq. 29) describes the classical CEV model (Sec. 2).

Analogous to the previous cases, the transformation (2) and the fractional Itô's lemma goes Eq. 29] to:

\footnotetext{
${ }^{10}$ Cheridito 18 proves that for $\left.H \in\right] 3 / 4,1\left[\right.$ the filtration generated by $M_{t}^{\beta, \gamma, H}$ is equivalent to a classical Brownian motion; i.e, a semi-martingale.
} 


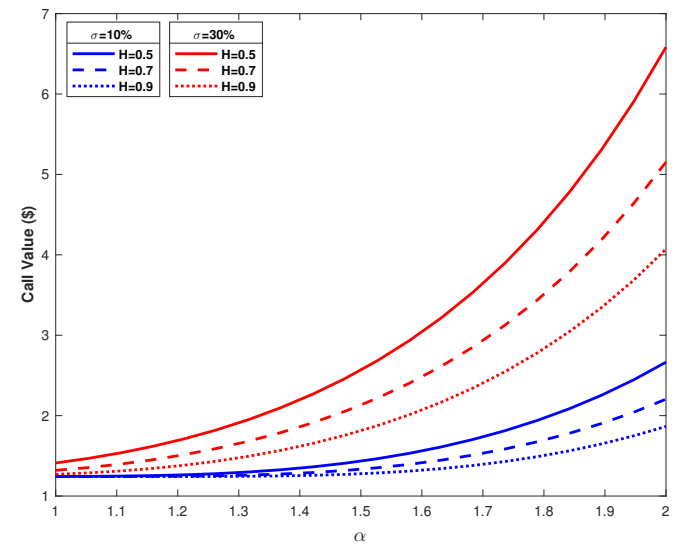

(a) $\mathrm{T}=0.25$

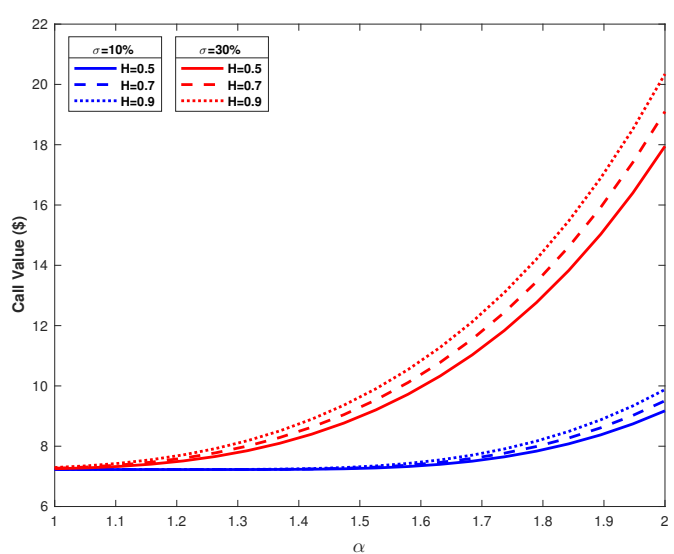

(c) $\mathrm{T}=1.5$

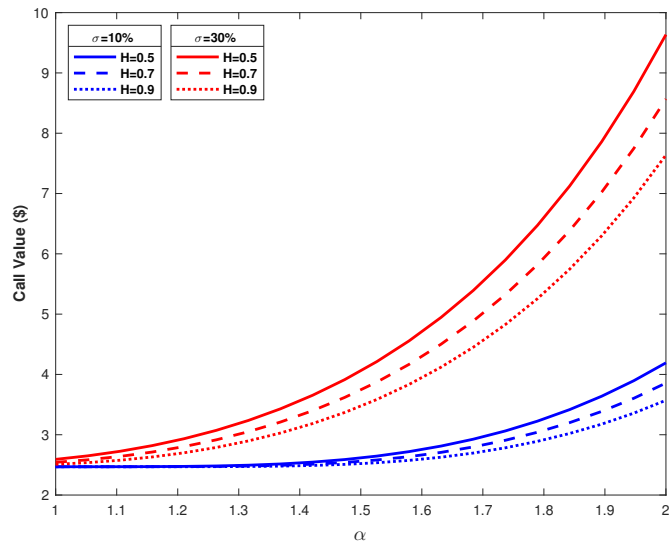

(b) $\mathrm{T}=0.5$

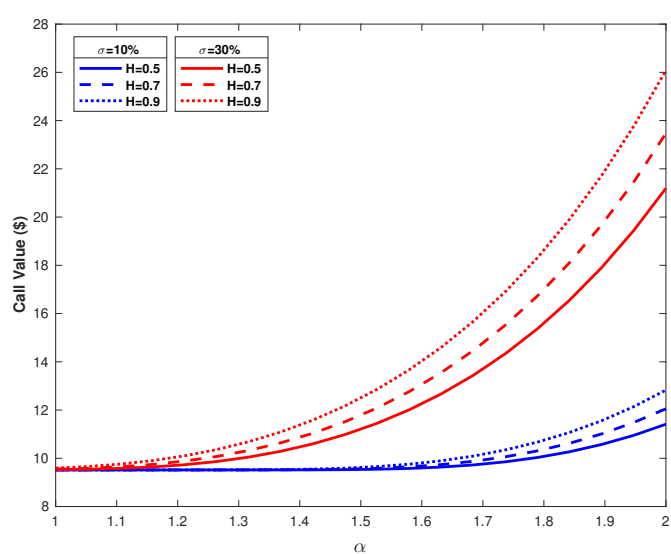

(d) $\mathrm{T}=2$

Figure 1: Fractional CEV formula for a European Call with $\sigma=\{10 \% ; 30 \%\}$ and $H=\{0.5 ; 0.7 ; 0.9\}$ and different maturities in function of the elasticity parameter, fixing $S_{0}=E=100$ and $r=5 \%$. 


$$
\mathrm{d} x=(2-\alpha)\left[r x+\left(\frac{1}{2} \beta+\gamma H t^{2 H-1}\right)(1-\alpha) \sigma^{2}\right] \mathrm{d} t+(2-\alpha) \sigma \sqrt{x}\left(\beta \mathrm{d} B_{t}+\gamma \mathrm{d} B_{t}^{H}\right)
$$

Since that $B_{t}=B_{t}^{1 / 2}$, the fractional Fokker-Planck equation for the above process is:

$$
\frac{\partial P_{M}}{\partial t}=\frac{1}{2} \frac{\partial^{2}}{\partial x^{2}}\left[\left(\beta+2 \gamma H t^{2 H-1}\right)(2-\alpha)^{2} \sigma^{2} x P\right]-\frac{\partial}{\partial x}\left\{(2-\alpha)\left[r x+\left(\beta+2 \gamma H t^{2 H-1}\right) \frac{(1-\alpha)}{2} \sigma^{2}\right] P\right\}
$$

Setting:

$$
\begin{aligned}
A^{\prime}(\tau) & =\gamma A(\tau)-\frac{a}{b} \beta \\
C^{\prime}(\tau) & =\gamma C(\tau)-\frac{a}{b} \beta \\
\theta^{\prime} & =\frac{C^{\prime}(\tau)}{A^{\prime}(\tau)}
\end{aligned}
$$

the relation 30 becomes:

$$
\frac{\partial P_{M}}{\partial \tau}=\frac{\partial^{2}}{\partial x^{2}}\left[A^{\prime}(\tau) x P\right]+\frac{\partial}{\partial x}\left\{(2-\alpha)\left[x-C^{\prime}(\tau)\right] P\right\}
$$

where $\tau A, C$ are given by Eqs. (18)- 20 .

Given that $\theta^{\prime}=\theta=c / a$ (constant), a solution for (31) is obtained through the Feller's lemma with time-varying coefficients

$$
P_{M}\left(x, \tau \mid x_{0}, 0\right)=\frac{1}{\phi^{\prime}(\tau)}\left(\frac{x \mathrm{e}^{\tau}}{x_{0}}\right)^{\frac{c-a}{2 a}} \exp \left[-\frac{\left(x+x_{0} \mathrm{e}^{-\tau}\right)}{\phi^{\prime}(\tau)}\right] I_{1-c / a}\left[\frac{2}{\phi^{\prime}(\tau)} \sqrt{\mathrm{e}^{-\tau} x_{0} x}\right]
$$

and

$$
\begin{aligned}
\phi(\tau) & =-\frac{a}{b} \int_{0}^{\tau} \gamma\left[2 H\left(\frac{s-\tau}{b}\right)^{2 H-1}-\beta\right] \mathrm{e}^{-s} \mathrm{~d} s \\
& =\gamma \frac{a}{2 H+1}\left(-\frac{\tau}{b}\right)^{2 H}\left[2 H+1+\mathrm{e}^{-\frac{1}{2} \tau}(-\tau)^{-H} M_{H, H+1 / 2}(-\tau)\right]+\beta \frac{a}{b}\left(\mathrm{e}^{-\tau}-1\right)
\end{aligned}
$$

Later, the transition probability density function for $S_{T}$ conditional to $S_{0}$, under a mixed-fractional regime is given by:

$$
P_{M}\left(S_{T}, T \mid S_{0}, 0\right)=(2-\alpha) k_{M}^{\frac{1}{2-\alpha}}\left(y w^{1-2 \alpha}\right)^{\frac{1}{2(2-\alpha)}} \mathrm{e}^{-y-w} I_{1 /(2-\alpha)}(2 \sqrt{y w})
$$

being :

$$
\begin{aligned}
k_{M} & =\left[\phi^{\prime}(T)\right]^{-1}, \\
y_{M} & =k_{M} S_{0}^{2-\alpha} \mathrm{e}^{r(2-\alpha) T} \\
w_{M} & =k_{M} S_{T}^{2-\alpha}
\end{aligned}
$$

with, 


$$
\begin{aligned}
\phi^{\prime}(T) & =\frac{a}{2 H+1} T^{2 H}\left[2 H+1+\mathrm{e}^{\frac{1}{2} b T}(b T)^{-H} M_{H, H+1 / 2}(b T)\right]+\beta \frac{a}{b}\left(\mathrm{e}^{-\tau}-1\right) \\
& =\gamma \phi(T)+\beta \frac{a}{b}\left(\mathrm{e}^{-\tau}-1\right)
\end{aligned}
$$

Using the argument provided in the previous sections, the European Call price, at time $t=0$ under the mixed-fractional CEV framework is computed by:

$$
C_{M}\left(S_{0}, 0\right)=S_{0} Q\left(2 z_{M} ; 2+\frac{2}{2-\alpha}, 2 y_{M}\right)-E \mathrm{e}^{-r T}\left[1-Q\left(2 y_{M} ; \frac{2}{2-\alpha}, 2 z_{M}\right)\right]
$$

where $z_{H}(t)=k_{H}(t) E^{2-\alpha}$.

Eq. (35) turns into the pure fractional CEV case (Eq. (26) if $(\beta, \gamma)=(1,0)$, and becomes the classical CEV model if $(\beta, \gamma)=(1,0)$ or $(\beta, \gamma, H)=(0,1,1 / 2)$.

Using the results computed in the Eqs. (13), 114, 27) and 28); at the limit case $\alpha \rightarrow 2$, Eq. 35 , tends to:

$$
\lim _{\alpha \rightarrow 2^{-}} C_{M}\left(S_{0}, 0\right)=S_{0} N\left(d_{1}^{M}\right)-E \mathrm{e}^{-r T} N\left(d_{2}^{M}\right)
$$

with

$$
\begin{aligned}
& d_{1}^{M}=\frac{\ln \left(\frac{S_{0}}{E}\right)+r T+\frac{1}{2} \beta \sigma^{2} T+\frac{1}{2} \gamma \sigma^{2} T^{2 H}}{\sigma \sqrt{\beta T+\gamma T^{2 H}}} \\
& d_{2}^{M}=\frac{\ln \left(\frac{S_{0}}{E}\right)+r T-\frac{1}{2} \beta \sigma^{2} T-\frac{1}{2} \gamma \sigma^{2} T^{2 H}}{\sigma \sqrt{\beta T+\gamma T^{2 H}}}
\end{aligned}
$$

which is the mixed-fractional Black-Scholes pricing formula (for instance, see 24] with $\beta=1$ and $\gamma=1$ ); keeping the convergence between the CEV and Black-Scholes.

Fig. 2 display the value for a European Call under the mixed-fractional CEV model, setting the pair of coefficients $\beta$ and $\gamma$ as $(\beta, \gamma)=(1,1)$ and $(\beta, \gamma)=(0,1)$ (blue and red respectively), with the aim of to compare the mixed fractional and pure fractional cases. We consider two maturities $\mathrm{T}=0.5$ (2a) and $\mathrm{T}=1.5(2 \mathrm{~b})$ and $H \in\{0.5 ; 0.7 ; 0.9\}$. In both subplots the classical CEV pricing is represented by the red-solid-line. We can observe that the mixed-fractional price is higher than the both classical and fractional price. As in the pure fractional case, for $\mathrm{T}<1$ the price decreases if $\mathrm{H}$ tends to one, and increases for $\mathrm{T}>1$ and $\mathrm{H}$ moves to one.

Fig. (3) addresses the computational cost, measured as CPU time, of the mixed and pure fractional models. For $\alpha \rightarrow 2$, the formula becomes extremely expensive. Nevertheless, there are no significative differences in terms of cost between the classical and fractional approaches.

\section{Greeks}

For to analyze the sensitivities of the pricing formula as function of the parameters of the model, we compare the Greeks of both classical, fractional and mixed-fractional CEV models.

The most common sensitivities are related to the price, maturity, volatility and interest rate. We use the results given in Ref. [44] for $\Delta, \Gamma, \nu, \Theta$ and $\rho$ Greeks under the classical CEV model and here we carefully extent it to the fractional cases.

\subsection{Delta}

$$
\begin{aligned}
\Delta & =\frac{\partial C}{\partial S} \\
& =Q\left(2 z, 2+\frac{2}{2-\alpha}, 2 y\right)+\frac{2 y(2-\alpha)}{S}\left[S f\left(2 z ; 4+\frac{2}{2-\beta}, 2 y\right)-E \mathrm{e}^{-r T} f\left(2 y ; \frac{2}{2-\beta}, 2 z\right)\right](36)
\end{aligned}
$$




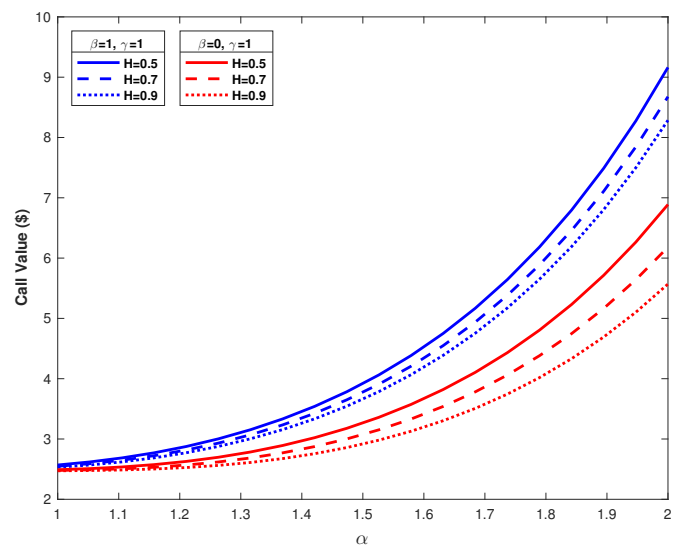

(a) $\mathrm{T}=0.5$

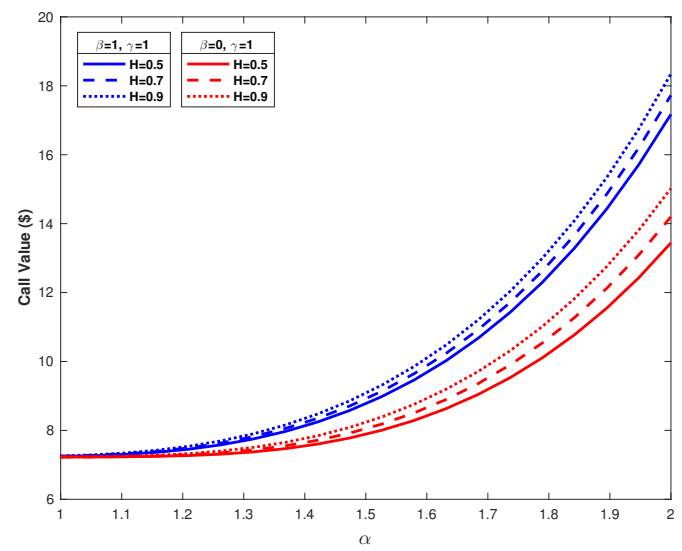

(b) $\mathrm{T}=1.5$

Figure 2: Mixed-fractional and fractional CEV formula using $S_{0}=E=100, r=5 \%$ and $\sigma=20 \%$

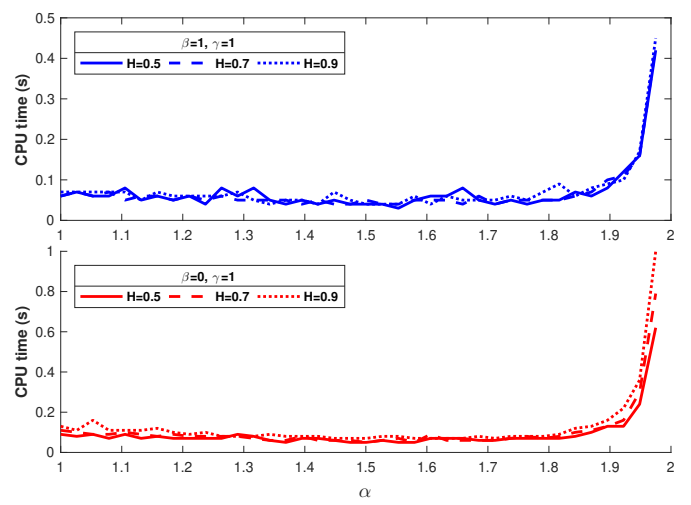

(a) $\mathrm{T}=0.5$

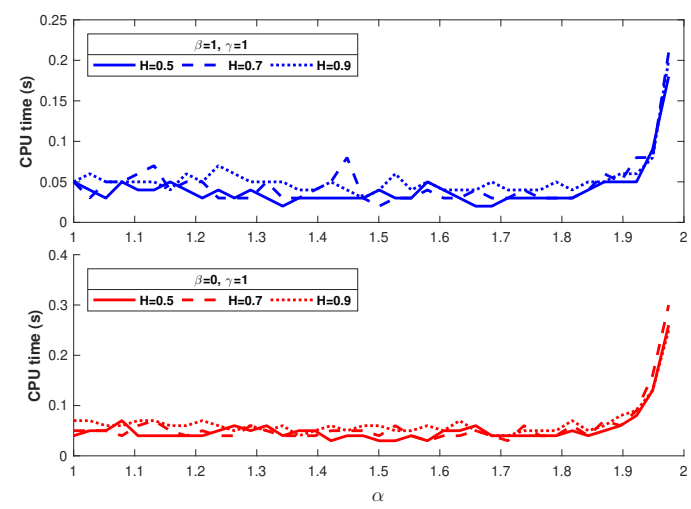

(b) $\mathrm{T}=1.5$

Figure 3: CPU times for the computation of the Mixed-fractional and fractional CEV formula using $S_{0}=E=100, r=5 \%$ and $\sigma=20 \%$ 


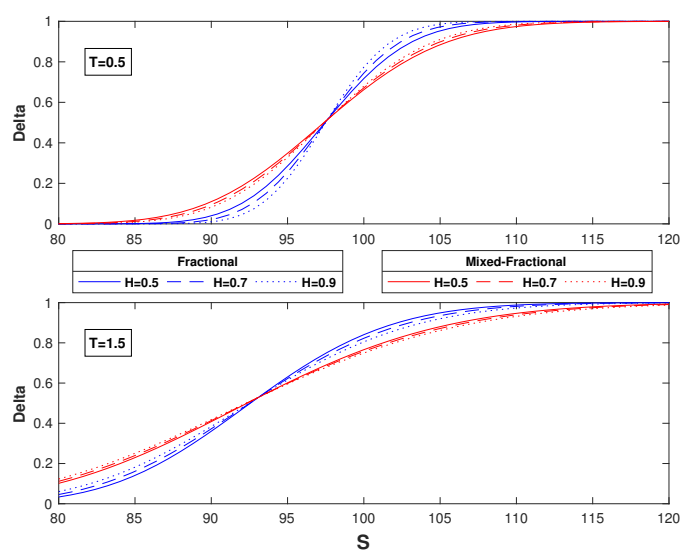

(a) $S \in[80,120]$ and $\alpha=1.5$

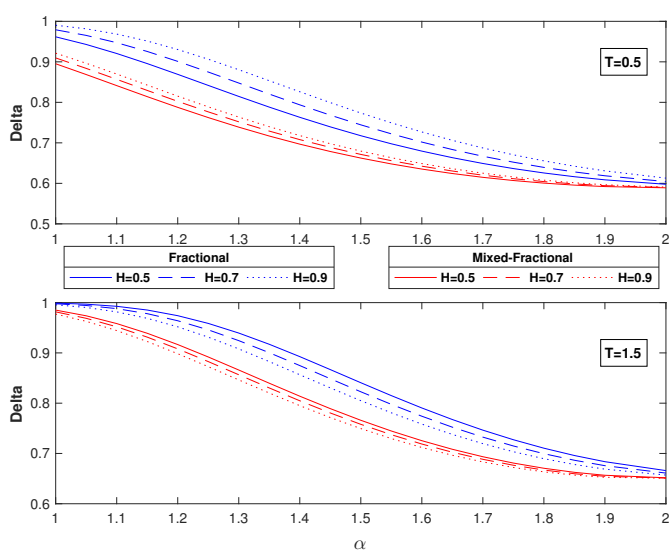

(b) $\mathrm{S}=100$ and $\alpha \in[1,2]$

Figure 4: Delta for fractional (blue) and mixed-fractional (red) models setting $E=100, \sigma=20 \%, r=5 \%$ and $\gamma=\beta=1$.

where $f$ is the non-central- $\chi^{2}$ PDF defined in 10 .

For the fractional case, we have:

$$
\begin{aligned}
\Delta_{H}= & \frac{\partial C_{H}}{\partial S} \\
= & Q\left(2 z_{H}, 2+\frac{2}{2-\alpha}, 2 y_{H}\right) \\
& +\frac{2 y_{H}(2-\alpha)}{S}\left[S f\left(2 z_{H} ; 4+\frac{2}{2-\beta}, 2 y_{H}\right)-E \mathrm{e}^{-r T} f\left(2 y_{H} ; \frac{2}{2-\beta}, 2 z_{H}\right)\right]
\end{aligned}
$$

and in the mixed-fractional:

$$
\begin{aligned}
\Delta_{M}= & \frac{\partial C_{M}}{\partial S} \\
= & Q\left(2 z_{M}, 2+\frac{2}{2-\alpha}, 2 y_{M}\right) \\
& +\frac{2 y_{M}(2-\alpha)}{S}\left[S f\left(2 z_{M} ; 4+\frac{2}{2-\beta}, 2 y_{M}\right)-E \mathrm{e}^{-r T} f\left(2 y_{M} ; \frac{2}{2-\beta}, 2 z_{M}\right)\right]
\end{aligned}
$$

The charts at the Figure 4 show the behavior of the $\Delta, \Delta_{H}$ and $\Delta_{M}$ varying the spot price $4 \mathrm{at}$ and elasticity (4b) for maturities below and above the unity. The solid blue line corresponds to the classical CEV model.

\subsection{Gamma}

The eqs. 39,40 and 41 provide the Gamma sensitivity for standard, fractional and mixed-fractional CEV, respectively, plotting it at Fig. 5 


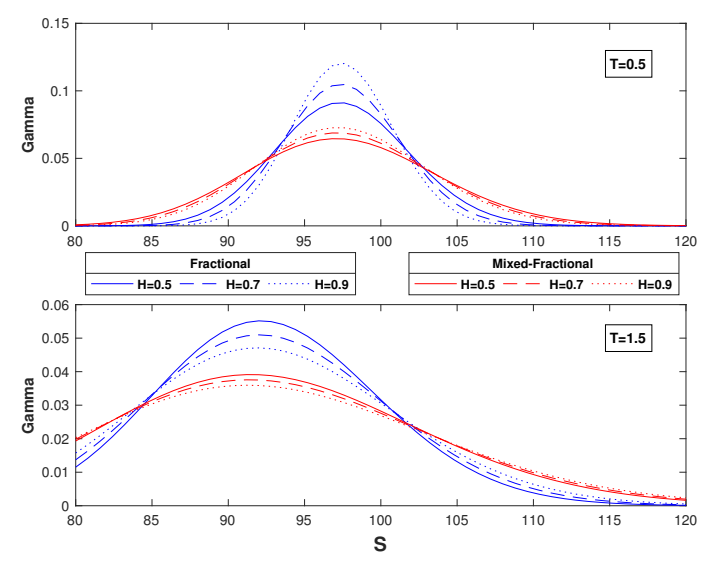

(a) $S \in[80,120]$ and $\alpha=1.5$

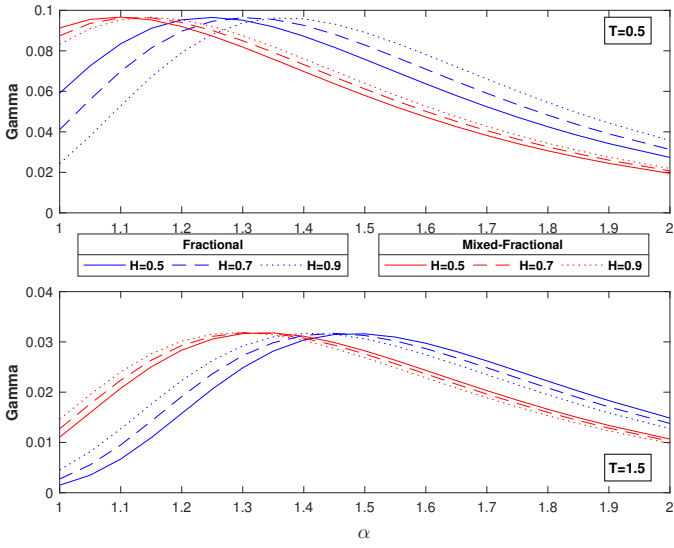

(b) $\mathrm{S}=100$ and $\alpha \in[1,2]$

Figure 5: Gamma for fractional (blue) and mixed-fractional (red) models setting $E=100, \sigma=20 \%$, $r=5 \%$ and $\gamma=\beta=1$.

$$
\begin{aligned}
\Gamma= & \frac{\partial^{2} C}{\partial S^{2}} \\
= & \frac{\partial}{\partial S} \Delta \\
= & \frac{2 y(2-\alpha)^{2}}{S}\left\{\left[\frac{(3-\alpha)}{2-\alpha}-y\right] f\left(2 z ; 4+\frac{2}{2-\beta}, 2 y\right)+y f\left(2 z ; 6+\frac{2}{2-\beta}, 2 y\right)\right\} \\
& +\frac{2 y(2-\alpha)^{2}}{S^{2}} E \mathrm{e}^{-r T}\left[y f\left(2 y ; \frac{2}{2-\beta}, 2 z\right)-z f\left(2 y ; 2+\frac{2}{2-\beta}, 2 z\right)\right]
\end{aligned}
$$

$$
\begin{aligned}
\Gamma_{H}= & \frac{\partial^{2} C_{H}}{\partial S^{2}} \\
= & \frac{2 y_{H}(2-\alpha)^{2}}{S}\left\{\left[\frac{(3-\alpha)}{2-\alpha}-y_{H}\right] f\left(2 z_{H} ; 4+\frac{2}{2-\beta}, 2 y_{H}\right)+y_{H} f\left(2 z_{H} ; 6+\frac{2}{2-\beta}, 2 y_{H}\right)\right\} \\
& +\frac{2 y_{H}(2-\alpha)^{2}}{S^{2}} E \mathrm{e}^{-r T}\left[y_{H} f\left(2 y_{H} ; \frac{2}{2-\beta}, 2 z_{H}\right)-z_{H} f\left(2 y_{H} ; 2+\frac{2}{2-\beta}, 2 z_{H}\right)\right]
\end{aligned}
$$

$$
\begin{aligned}
\Gamma_{M}= & \frac{\partial^{2} C_{M}}{\partial S^{2}} \\
= & \frac{2 y_{M}(2-\alpha)^{2}}{S}\left\{\left[\frac{(3-\alpha)}{2-\alpha}-y_{M}\right] f\left(2 z_{M} ; 4+\frac{2}{2-\beta}, 2 y_{M}\right)+y_{M} f\left(2 z_{M} ; 6+\frac{2}{2-\beta}, 2 y_{M}\right)\right\} \\
& +\frac{2 y_{M}(2-\alpha)^{2}}{S^{2}} E \mathrm{e}^{-r T}\left[y_{M} f\left(2 y_{M} ; \frac{2}{2-\beta}, 2 z_{M}\right)-z_{M} f\left(2 z_{M} ; 2+\frac{2}{2-\beta}, 2 y_{M}\right)\right]
\end{aligned}
$$

\section{$5.3 \quad$ Vega}

The partial derivative with respect to the volatility is called Vega (commonly represented by the greek letter $\nu$ ). In the $\mathrm{CEV}$ model, the volatility is a function of both $S$ and the parameter $\sigma$, defined by $\boldsymbol{\sigma}^{2}=\sigma S^{\frac{\alpha-2}{2}}$. Then the Vega for the CEV model is: 


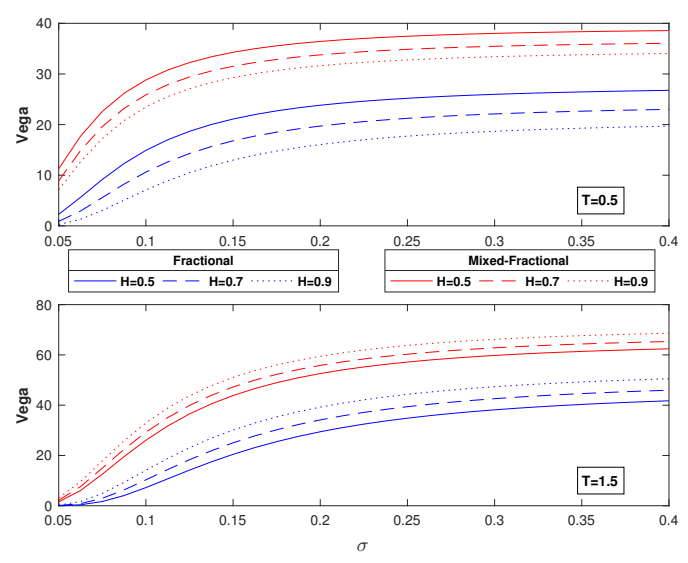

(a) $\sigma \in[0.05,0.4]$ and $\alpha=1.5$

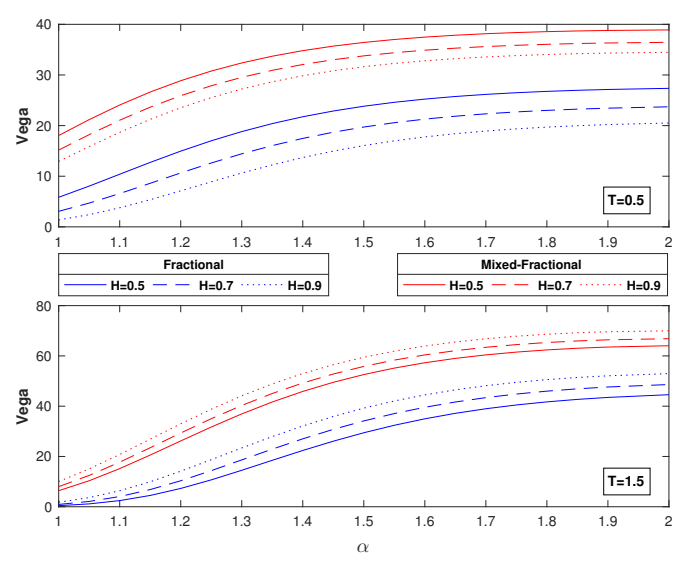

(b) $\sigma=20 \%$ and $\alpha \in[1,2]$

Figure 6: Vega for fractional (blue) and mixed-fractional (red) models setting $S=E=100, r=5 \%$ and $\gamma=\beta=1$.

$$
\begin{aligned}
\nu & =\frac{\partial C}{\partial \boldsymbol{\sigma}} ; \quad \sigma^{2}=\boldsymbol{\sigma}^{2} S^{2-\alpha} \\
& =\frac{\partial C}{\partial \sigma} \frac{\partial \sigma}{\partial \boldsymbol{\sigma}} \\
& =\left[\frac{\partial C}{\partial(2 y)} \frac{\partial(2 y)}{\partial k}\right] \frac{(-2 k)}{\sigma} S^{\frac{2-\alpha}{2}} \\
& =\frac{-4 y}{\boldsymbol{\sigma}}\left[S f\left(2 z, 4+\frac{2}{2-2 \alpha}, 2 y\right)-E \mathrm{e}^{-r T} f\left(2 y, \frac{2}{2-\alpha}, 2 z\right)\right]
\end{aligned}
$$

For the fractional and mixed-fractional models, we have:

$$
\begin{aligned}
\nu_{H} & =\frac{\partial C_{H}}{\partial \boldsymbol{\sigma}} \\
& =\left[\frac{\partial C_{H}}{\partial\left(2 y_{H}\right)} \frac{\partial\left(2 y_{H}\right)}{\partial k_{H}}\right] \frac{\left(-2 k_{H}\right)}{\sigma} S^{\frac{2-\alpha}{2}} \\
& =\frac{-4 y_{H}}{\sigma}\left[S f\left(2 z_{H}, 4+\frac{2}{2-2 \alpha}, 2 y_{H}\right)-E \mathrm{e}^{-r T} f\left(2 y_{H}, \frac{2}{2-\alpha}, 2 z_{H}\right)\right] \\
\nu_{M} & =\frac{\partial C_{M}}{\partial \boldsymbol{\sigma}} \\
& =\left[\frac{\partial C_{M}}{\partial\left(2 y_{M}\right)} \frac{\partial\left(2 y_{M}\right)}{\partial k_{M}}\right] \frac{\left(-2 k_{M}\right)}{\sigma} S^{\frac{2-\alpha}{2}} \\
& =\frac{-4 y_{M}}{\sigma}\left[S f\left(2 z_{M}, 4+\frac{2}{2-2 \alpha}, 2 y_{M}\right)-E \mathrm{e}^{-r T} f\left(2 y_{M}, \frac{2}{2-\alpha}, 2 z_{M}\right)\right]
\end{aligned}
$$

The graphics in Fig. 6 display the Vega for the fractional and mixed-fractional models. The standard CEV Vega is plotted by the solid-blue line.

\subsection{Theta}

The change rate of the option price with respect to the maturity $T$ is noted by the greek $\Theta$, and is computed for the CEV model at the Eq. 45 for the fractional CEV at Eq. 46 and for the mixedfractional CEV at Eq. 47. Fig. 7 draw the shape of $\Theta_{H}$ and $\Theta_{M}$ under different values of $\mathrm{T}$ (7a) and alpha $7 \mathrm{bb}$. 


$$
\begin{aligned}
\Theta= & \frac{\partial C}{\partial T} \\
= & S \frac{\partial Q(2 z, 2+2 /(2-\alpha), 2 y)}{\partial(2 y)} \frac{\partial(2 y)}{\partial T}+r E \mathrm{e}^{-r T}[1-Q(2 y, 2 /(2-\alpha), 2 z)] \\
& +E \mathrm{e}^{-r T} \frac{\partial Q(2 y, 2 /(2-\alpha), 2 z)}{\partial(2 y)} \frac{\partial(2 y)}{\partial T} \\
= & -\frac{2 y r(2-\alpha)}{\mathrm{e}^{r(2-\alpha) T}-1}\left[S f\left(2 z, 4+\frac{2}{2-\alpha}, 2 y\right)-E \mathrm{e}^{-r T} f\left(2 y, \frac{2}{2-\alpha}, 2 z\right)\right] \\
& +r E \mathrm{e}^{-r T}[1-Q(2 y, 2 /(2-\alpha), 2 z)]
\end{aligned}
$$

$$
\begin{aligned}
\Theta_{H}= & \frac{\partial C_{H}}{\partial T} \\
= & S \frac{\partial Q\left(2 z_{H}, 2+2 /(2-\alpha), 2 y_{H}\right)}{\partial\left(2 y_{H}\right)} \frac{\partial\left(2 y_{H}\right)}{\partial T}+r E \mathrm{e}^{-r T}\left[1-Q\left(2 y_{H}, 2 /(2-\alpha), 2 z_{H}\right)\right] \\
& +E \mathrm{e}^{-r T} \frac{\partial Q\left(2 y_{H}, 2 /(2-\alpha), 2 z_{H}\right)}{\partial\left(2 y_{H}\right)} \frac{\partial\left(2 y_{H}\right)}{\partial T} \\
= & -2 y_{H} H T^{2 H-1} \frac{\sigma^{2}(2-\alpha)^{2}}{\phi(T)}\left[S f\left(2 z_{H}, 4+\frac{2}{2-\alpha}, 2 y_{H}\right)-E \mathrm{e}^{-r T} f\left(2 y_{H}, \frac{2}{2-\alpha}, 2 z_{H}\right)\right] \\
& +r E \mathrm{e}^{-r T}\left[1-Q\left(2 y_{H}, 2 /(2-\alpha), 2 z_{H}\right)\right]
\end{aligned}
$$

$$
\begin{aligned}
\Theta_{M}= & \frac{\partial C_{M}}{\partial T} \\
= & -2 y_{M} \frac{\sigma^{2}(2-\alpha)^{2}}{\phi^{\prime}(T)}\left[\gamma H T^{2 H-1}+\frac{\beta}{2}\right]\left[S f\left(2 z_{M}, 4+\frac{2}{2-\alpha}, 2 y_{M}\right)\right. \\
& \left.-E \mathrm{e}^{-r T} f\left(2 y_{M}, \frac{2}{2-\alpha}, 2 z_{M}\right)\right]+r E \mathrm{e}^{-r T}\left[1-Q\left(2 y_{M}, 2 /(2-\alpha), 2 z_{M}\right)\right]
\end{aligned}
$$

\section{$5.5 \quad$ Rho}

Finally, the sensitivity respect to the risk-free-interest rate, for the CEV model and its extensions (fractional and mixed-fractional), are shown at Fig. 8 and explicitly computed at the eqs. $48.49,50$.

$$
\begin{aligned}
\rho= & \frac{\partial C}{\partial r} \\
= & S \frac{\partial Q(2 z, 2+2 /(2-\alpha), 2 y)}{\partial(2 y)} \frac{\partial(2 y)}{\partial r}+T E \mathrm{e}^{-r T}[1-Q(2 y, 2 /(2-\alpha), 2 z)] \\
& +E \mathrm{e}^{-r T} \frac{\partial Q(2 y, 2 /(2-\alpha), 2 z)}{\partial(2 y)} \frac{\partial(2 y)}{\partial T} \\
= & 2 y\left[\frac{1}{r}-\frac{(2-\alpha) T}{\mathrm{e}^{r(2-\alpha) T}-1}\right]\left[S f\left(2 z, 4+\frac{2}{2-\alpha}, 2 y\right)-E \mathrm{e}^{-r T}\left(2 y, \frac{2}{2-\alpha}, 2 z\right)\right] \\
& +T E \mathrm{e}^{-r T}[1-Q(2 y, 2 /(2-\alpha), 2 z)]
\end{aligned}
$$




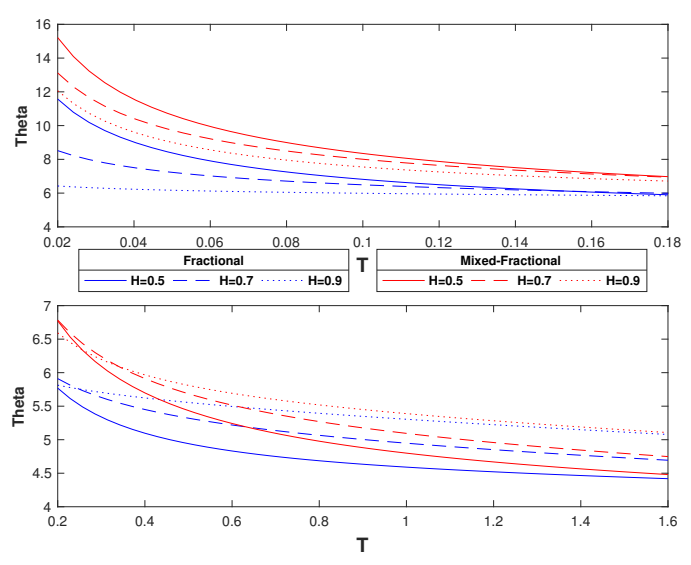

(a) $T \in[0.02,1.6]$ and $\alpha=1.5$

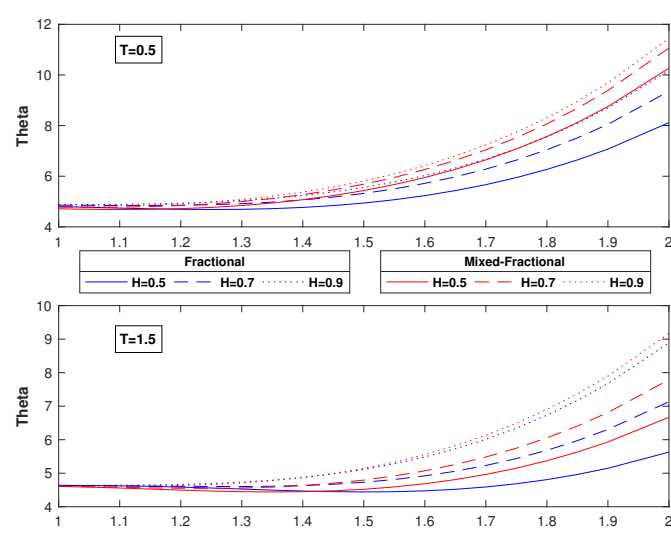

(b) $\alpha \in[1,2]$

Figure 7: Theta for fractional (blue) and mixed-fractional (red) models setting $S=E=100, r=5 \%$, $\sigma=20 \%$ and $\gamma=\beta=1$.

$$
\begin{aligned}
\rho_{H}= & \frac{\partial C_{H}}{\partial r} \\
= & S \frac{\partial Q\left(2 z_{H}, 2+2 /(2-\alpha), 2 y_{H}\right)}{\partial\left(2 y_{H}\right)} \frac{\partial(2 y)}{\partial r}+T E \mathrm{e}^{-r T}\left[1-Q\left(2 y_{H}, 2 /(2-\alpha), 2 z_{H}\right)\right] \\
& +E \mathrm{e}^{-r T} \frac{\partial Q\left(2 y_{H}, 2 /(2-\alpha), 2 z_{H}\right)}{\partial\left(2 y_{H}\right)} \frac{\partial\left(2 y_{H}\right)}{\partial T} \\
= & 2 y_{H}\left[\frac{2 H}{r}-\frac{H(2-\alpha)^{2} \sigma^{2} T^{2 H}}{r \phi(T)}\right]\left[S f\left(2 z_{H}, 4+\frac{2}{2-\alpha}, 2 y_{H}\right)-E \mathrm{e}^{-r T}\left(2 y_{H}, \frac{2}{2-\alpha}, 2 z_{H}\right)\right] \\
& +T E \mathrm{e}^{-r T}\left[1-Q\left(2 y_{H}, 2 /(2-\alpha), 2 z_{H}\right)\right] \\
\rho_{M}= & \frac{\partial C_{M}}{\partial r} \\
& \frac{2 y_{M}}{\phi^{\prime}(T) r}\left\{\gamma\left[2 H \phi(T)-H(2-\alpha)^{2} \sigma^{2} T^{2 H}\right]+\beta \frac{(2-\alpha) \sigma^{2}}{2 r}\left[\mathrm{e}^{r(2-\alpha) T}-1-r(2-\alpha) T\right]\right\} \\
& \times\left[S f\left(2 z_{M}, 4+\frac{2}{2-\alpha}, 2 y_{M}\right)-E \mathrm{e}^{-r T}\left(2 y_{M}, \frac{2}{2-\alpha}, 2 z_{M}\right)\right] \\
& +T E \mathrm{e}^{-r T}\left[1-Q\left(2 y_{M}, 2 /(2-\alpha), 2 z_{M}\right)\right]
\end{aligned}
$$

\section{Summary}

In this paper, the constant elasticity of variance model is studied, adding a fractal feature to the Brownian motion to address the long-memory in financial markets. Besides, to deal with the non-arbitrage issue under pure fractional regimes, a mixed fractional CEV model is developed.

Then, using the fractional Itô calculus and the fractional generalization of the Fokker-Planck equations, an analytical and compact option pricing scheme for a European Call, based on the complementary noncentral-chi-squared density function and the M-Whittaker function, is provided for both approaches. The convergence to both the classical CEV model and the fractional \& mixed-fractional Black Scholes formula are shown for the limit cases $H \rightarrow 1 / 2$ and $\alpha \rightarrow 2$, respectively, and then the proposed fractional extensions could be interpreted as a generalization of the classical CEV and Black-Scholes model. Besides, 


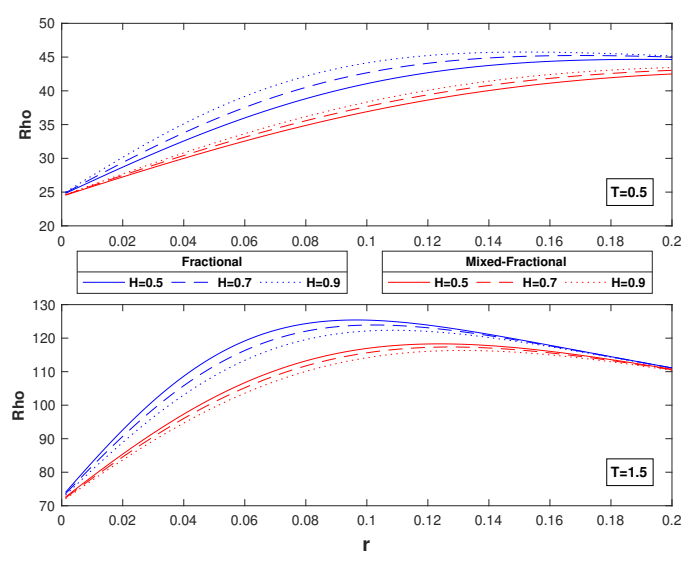

(a) $r \in[0.01,0.2]$ and $\alpha=1.5$

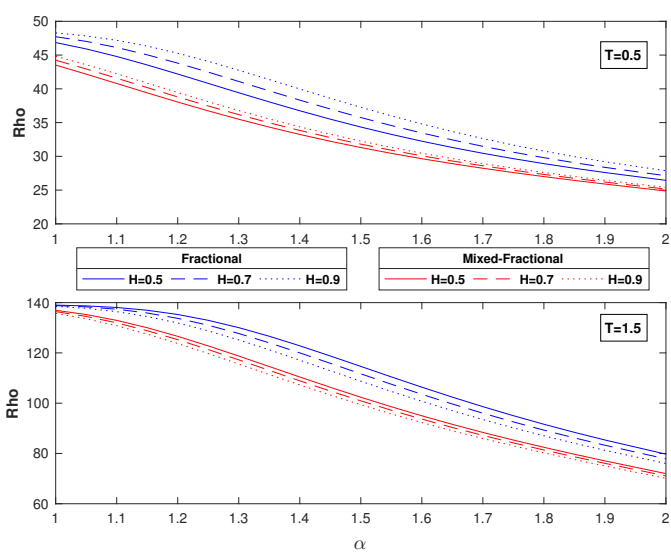

(b) $r=5 \%$ and $\alpha \in[1,2]$

Figure 8: Rho for fractional (blue) and mixed-fractional (red) models setting $S=E=100, \sigma=20 \%$ and $\gamma=\beta=1$.

the Greeks are computed showing their behavior under different values of the Hurst exponent, considering maturities lower and greater than one.

Since the added terms on the call formula in both fractional models, in relation to the classical CEV, doesn't have a dependency on the strike price, the fractional and mixed-fractional CEV keep the capability to address the smile-skew issue.

\section{Acknowledgements}

I thank FIAS for financial support and both Dr. Nils Bertschinger and Dr. Marcelo Villena for helpful discussions.

\section{References}

[1] Fischer Black and Myron Scholes. The pricing of options and corporate liabilities. Journal of Political Economy, 81(3):637-654, 1973.

[2] Andrew W. Lo. Long-term memory in stock market prices. Econometrica, 59(5):1279-1313, 1991.

[3] Walter Willinger, Murad Taqqu, and Vadim Teverovsky. Stock market prices and long-range dependence. Finance and stochastics, 3(1):1-13, 1999.

[4] Shibley Sadique and Param Silvapulle. Long-term memory in stock market returns: international evidence. International Journal of Finance \& Economics, 6(1):59-67, 2001.

[5] Daniel O Cajueiro and Benjamin M Tabak. The hurst exponent over time: testing the assertion that emerging markets are becoming more efficient. Physica A: Statistical Mechanics and its Applications, 336(3-4):521-537, 2004.

[6] Nigel Cutland, P. Ekkehard Kopp, and Walter Willinger. Stock price returns and the Joseph effect: a fractional version of the Black-Scholes model. In Seminar on stochastic analysis, random fields and applications, pages 327-351. Springer, 1995.

[7] W Dai and CC Heyde. Itô's formula with respect to fractional brownian motion and its application. International Journal of Stochastic Analysis, 9(4):439-448, 1996.

[8] Benoit B. Mandelbrot and John W. Van Ness. Fractional Brownian motions, fractional noises and applications. SIAM review, 10(4):422-437, 1968.

[9] Benoit Mandelbrot. Gaussian self-affinity and fractals: Globality, the earth, $1 / f$ noise, and $R / S$, volume 8. Springer Science \& Business Media, NY, USA, 2002. 
[10] Francesca Biagini, Yaozhong Hu, Bernt Øksendal, and Tusheng Zhang. Stochastic calculus for fractional Brownian motion and applications. Springer Science \& Business Media, 2008.

[11] Yaozhong $\mathrm{Hu}$ and Bernt Øksendal. Fractional white noise calculus and applications to finance. Infinite dimensional analysis, quantum probability and related topics, 6(01):1-32, 2003.

[12] Ciprian Necula. Option pricing in a fractional Brownian motion environment. Working paper, Academy of Economic Studies, Bucharest, 2002.

[13] Tyrone Duncan, Yaozhong Hu, and Bozenna Pasik-Duncan. Stochastic calculus for fractional Brownian motion I. Theory. SIAM Journal on Control and Optimization, 38(2):582-612, 2000.

[14] David Nualart and Murad Taqqu. Wick-Itô formula for regular processes and applications to the Black and Scholes formula. Stochastics An International Journal of Probability and Stochastic Processes, 80(5):477-487, 2008.

[15] Patrick Cheridito. Arbitrage in fractional brownian motion models. Finance and Stochastics, $7(4): 533-553,2003$.

[16] Tomas Björk and Henrik Hult. A note on Wick products and the fractional Black-Scholes model. Finance and Stochastics, 9(2):197-209, 2005.

[17] S. Rostek and R. Schöbel. A note on the use of fractional Brownian motion for financial modeling. Economic Modelling, 30:30-35, 2013.

[18] Patrick Cheridito. Mixed fractional Brownian motion. Bernoulli, 7(6):913-934, 2001.

[19] Mounir Zili. On the mixed fractional brownian motion. International Journal of Stochastic Analysis, 2006, 2006.

[20] Christoph Thäle. Further remarks on mixed fractional Brownian motion. Applied Mathematical Sciences, 38:1885-1901, 2009.

[21] Taras Androshchuk and Yuliya Mishura. Mixed Brownian-fractional Brownian model: absence of arbitrage and related topics. Stochastics An International Journal of Probability and Stochastic Processes, 78(5):281-300, 2006.

[22] Christian Bender, Tommi Sottinen, and Esko Valkeila. Arbitrage with fractional Brownian motion? Theory of stochastic processes, 13(29):23-34, 2007.

[23] Lin Sun. Pricing currency options in the mixed fractional Brownian motion. Physica A: Statistical Mechanics and its Applications, 392(16):3441-3458, 2013.

[24] C. Murwaningtyas, S. Kartiko, and H. Suryawan. European option pricing by using a mixed fractional Brownian motion. In Journal of Physics: Conference Series, volume 1097, page 012081. IOP Publishing, 2018.

[25] Emanuel Derman and Iraj Kani. Riding on a Smile. Risk, 7(2):32-39, 1994.

[26] Bruno Dupire. Pricing with a smile. Risk, 7(1):18-20, 1994.

[27] Mark Rubinstein. Nonparametric tests of alternative option pricing models using all reported trades and quotes on the 30 most active CBOE option classes from August 23, 1976 through August 31, 1978. The Journal of Finance, 40(2):455-480, 1985.

[28] Jens Carsten Jackwerth and Mark Rubinstein. Recovering probability distributions from option prices. The Journal of Finance, 51(5):1611-1631, 1996.

[29] Fisher Black. Studies of stock price volatility changes. In Proceedings of the Meetings of the American Statistical Association, Business and Economics Statistics Division, pages 177-181, August 1976.

[30] Andrew A. Christie. The stochastic behavior of common stock variances: Value, leverage and interest rate effects. Journal of Financial Economics, 10(4):407-432, 1982.

[31] Geert Bekaert and Guojun Wu. Asymmetric volatility and risk in equity markets. The Review of Financial Studies, 13(1):1-42, 2000. 
[32] Tim Bollerslev, Julia Litvinova, and George Tauchen. Leverage and volatility feedback effects in high-frequency data. Journal of Financial Econometrics, 4(3):353-384, 2006.

[33] John C. Cox. Notes on option pricing I: Constant elasticity of variance diffusions. Working paper, Stanford University, 1975.

[34] John C. Cox. The constant elasticity of variance option pricing model. The Journal of Portfolio Management, 23(5):15-17, 1996.

[35] Stan Beckers. The constant elasticity of variance model and its implications for option pricing. The Journal of Finance, 35(3):661-673, 1980.

[36] Min Chen. Equity volatility smile and skew under a CEV-based structural leverage model. Applied Mathematics $\&$ Information Sciences, 9(3):1095-1104, 2015.

[37] Shuang Xiao, Guo Li, and Yunjing Jia. Estimating the constant elasticity of variance model with data-driven markov chain monte carlo methods. Asia-Pacific Journal of Operational Research, 34(01):1740009, 2017.

[38] CF Lee, Ta-Peng Wu, and Ren-Raw Chen. The constant elasticity of variance models: New evidence from s\&p 500 index options. Review of Pacific Basin Financial Markets and Policies, 7(02):173-190, 2004.

[39] James D. MacBeth and Larry J. Merville. Tests of the Black-Scholes and Cox call option valuation models. The Journal of Finance, 35(2):285-301, 1980.

[40] Alan L. Tucker, David R. Peterson, and Elton Scott. Tests of the Black-Scholes and constant elasticity of variance currency call option valuation models. Journal of Financial Research, 11(3):201-214, 1988.

[41] V. K. Singh and N. Ahmad. Forecasting performance of constant elasticity of variance model: Empirical evidence from india. International Journal of Applied Economics and Finance, 5(1):8796, 2011.

[42] Mark Schroder. Computing the constant elasticity of variance option pricing formula. the Journal of Finance, 44(1):211-219, 1989.

[43] Shengliang Zhang, Hongqiang Yang, and Yu Yang. A multiquadric quasi-interpolations method for cev option pricing model. Journal of Computational and Applied Mathematics, 347:1-11, 2019.

[44] Manuela Larguinho, José Carlos Dias, and Carlos A. Braumann. On the computation of option prices and Greeks under the CEV model. Quantitative Finance, 13(6):907-917, 2013.

[45] Yajie Wang, Ximin Rong, and Hui Zhao. Optimal investment strategies for an insurer and a reinsurer with a jump diffusion risk process under the cev model. Journal of Computational and Applied Mathematics, 328:414-431, 2018.

[46] Weipeng Yuan and Shaoyong Lai. The cev model and its application to financial markets with volatility uncertainty. Journal of Computational and Applied Mathematics, 344:25-36, 2018.

[47] Weipeng Yuan and Shaoyong Lai. Family optimal investment strategy for a random household expenditure under the cev model. Journal of Computational and Applied Mathematics, 354:1-14, 2019.

[48] Ngai Hang Chan and Chi Tim Ng. Fractional constant elasticity of variance model. In Time Series and Related Topics, pages 149-164. Institute of Mathematical Statistics, 2006.

[49] William Feller. Two singular diffusion problems. Annals of mathematics, pages 173-182, 1951.

[50] Ying-Lin Hsu, T. I. Lin, and C. F. Lee. Constant elasticity of variance (CEV) option pricing model: Integration and detailed derivation. Mathematics and Computers in Simulation, 79(1):60-71, 2008.

[51] Donagh Horgan and Colin C Murphy. On the convergence of the chi square and noncentral chi square distributions to the normal distribution. IEEE Communications Letters, 17(12):2233-2236, 2013. 
[52] Ilkka Norros, Esko Valkeila, Jorma Virtamo, et al. An elementary approach to a Girsanov formula and other analytical results on fractional Brownian motions. Bernoulli, 5(4):571-587, 1999.

[53] Tommi Sottinen and Esko Valkeila. On arbitrage and replication in the fractional Black-Scholes pricing model. Statistics \& Decisions, 21(2/2003):93-108, 2003.

[54] Christian Bender. An Itô formula for generalized functionals of a fractional Brownian motion with arbitrary Hurst parameter. Stochastic Processes and their Applications, 104(1):81-106, 2003.

[55] Francesca Biagini, Bernt Øksendal, Agnès Sulem, and Naomi Wallner. An introduction to whitenoise theory and Malliavin calculus for fractional Brownian motion. In Proceedings of the Royal Society of London A: Mathematical, Physical and Engineering Sciences, volume 460, pages 347-372. The Royal Society, 2004.

[56] Ralf Metzler, E Barkai, and J Klafter. Deriving fractional fokker-planck equations from a generalised master equation. EPL (Europhysics Letters), 46(4):431, 1999.

[57] Ralf Metzler and Joseph Klafter. The random walk's guide to anomalous diffusion: a fractional dynamics approach. Physics reports, 339(1):1-77, 2000.

[58] Gazanfer Ünal. Fokker-Plank-Kolmogorov equation for fBm: Derivation and analytical solutions. In Mathematical physics, pages 53-60. World Scientific, 2007.

[59] Jaume Masoliver. Nonstationary feller process with time-varying coefficients. Physical Review E, 93(1):012122, 2016.

[60] Claudio Albanese, Giuseppe Campolieti, Peter Carr, and Alexander Lipton. Black-Scholes goes hypergeometric. Risk, 14(12):99-103, 2001.

[61] Rafael Mendoza-Arriaga and Vadim Linetsky. Pricing equity default swaps under the jump-to-default extended CEV model. Finance and Stochastics, 15(3):513-540, 2011.

[62] Sihun Jo, Minsuk Yang, and Geonwoo Kim. On convergence of laplace inversion for the american put option under the cev model. Journal of Computational and Applied Mathematics, 305:36-43, 2016.

[63] Edmund T Whittaker. An expression of certain known functions as generalized hypergeometric functions. Bulletin of the American Mathematical Society, 10(3):125-134, 1903.

[64] Herbert Buchholz. The confluent hypergeometric function: with special emphasis on its applications, volume 15 of Springer Tracks in Natural Philosophy. Springer, Berlin, 1969.

[65] Adri Olde Daalhuis. Confluent hypergeometric functions. In Frank Olver, Daniel Lozier, Ronald Boisvert, and Charles Clark, editors, NIST handbook of mathematical functions, volume 321-349, chapter 13. Cambridge University Press, 2010.

[66] Daniel Ocone. Malliavin's calculus and stochastic integral representations of functional of diffusion processes. Stochastics: An International Journal of Probability and Stochastic Processes, 12(3-4):161$185,1984$.

[67] Christian Bender and Robert Elliott. On the Clark-Ocone theorem for fractional Brownian motions with Hurst parameter bigger than a half. Stochastics and Stochastic Reports, 75(6):391-405, 2003.

[68] Xu Feng and Sun Quan. Pricing of option with power payoff driven by mixed fractional Brownian motion. In Proceedings of the 2010 Third International Conference on Business Intelligence and Financial Engineering, pages 170-173. IEEE Computer Society, 2010. 


\section{A Risk-neutral pricing in the fractional CEV model}

As pointed in 11, 12, the fractional Clark-Ocone theorem and quasi-expectations are used for to price a derivative under fractional Brownian motion. Here, we use the derivation of Hu and Øskendal [11] for to obtain a risk-neutral pricing at time $t=0$, but using a fractional CEV approach instead a fractional GBM.

Since the market is complete, at time $t$, a derivative $F$ is replicated by:

$$
F(t)=a(t) B(t)+b(t) S(t)
$$

where $a$ and $b$ are weights, $B$ is a money bank account (bond) which pays a continuously composed interest rate $r$ (risk-less interest rate; i.e, $\mathrm{d} B(t)=r B(t) \mathrm{d} t$ ) and $S$ is ruled by the Eq. 15

Later:

$$
\begin{aligned}
\mathrm{d} F(t) & =a(t) \mathrm{d} B(t)+b(t) \mathrm{d} S(t) \\
& =a(t) r B(t) \mathrm{d} t+b(t)\left[r S(t) \mathrm{d} t+\sigma(S(t))^{\alpha / 2} \mathrm{~d} B_{t}^{H}\right] \\
& =r[a(t) B(t)+b(t) S(t)] \mathrm{d} t+b(t) \sigma(S(t))^{\alpha / 2} \mathrm{~d} B_{t}^{H} \\
& =r F(t) \mathrm{d} t+b(t) \sigma S(t) \mathrm{d} B_{t}^{H}
\end{aligned}
$$

Multiplying 52 by $\mathrm{e}^{-r t}$ and integrating it from zero to $t$, we get:

$$
\mathrm{e}^{-r t} F(t)=F(0)+\int_{0}^{t} \mathrm{e}^{-r t} b(t) \sigma(S(t))^{\alpha / 2} \mathrm{~d} B_{t}^{H}
$$

On the other hand, the Clark-Ocone theorem for standard Brownian motions is given by [66]:

$$
G(t)=\mathbb{E}[G(T)]+\int_{0}^{T} D_{t} \mathbb{E}\left[G(t) \mid \mathcal{F}_{t}\right] \mathrm{d} B_{t}
$$

where $D_{t}$ is the Malliavin derivative and $\mathcal{F}_{t}$ is the natural filtration of Brownian motion. The fractional extension of the Eq. is provided by Refs. [11, 67]:

$$
G(t)=\mathbb{E}[G(T)]+\int_{0}^{T} D_{t} \mathbb{E}\left[G(t) \mid \mathcal{F}_{t}^{H}\right] \mathrm{d} B_{t}^{H}
$$

being $\mathcal{F}_{t}^{H}$ the $\sigma$-algebra generated by $B_{s}^{H}, s \leq t$. Put $G(t)=\mathrm{e}^{-r t} F(t)$ in 54

$$
\mathrm{e}^{-r t} F(t)=\mathbb{E}\left[\mathrm{e}^{-r t} F(T)\right]+\int_{0}^{T} D_{t} \mathbb{E}\left[\mathrm{e}^{-r t} F(t) \mid \mathcal{F}_{t}^{H}\right] \mathrm{d} B_{t}^{H}
$$

Comparing the expressions 5355 we arrive to the completeness of the market by:

$$
D_{t} \mathbb{E}\left[F(t) \mid \mathcal{F}_{t}^{H}\right]=b(t) \sigma(S(t))^{\alpha / 2}
$$

and

$$
F(0)=\mathbb{E}\left[\mathrm{e}^{-r t} F(T)\right]
$$


Then, at the initial time, the price of a derivative is computed discounted the expected value, as in the classical model driven by a Brownian motion.

For the mixed-fractional case, the extension is straightforward and is shown in [23, 68] for the mixed GBM. 